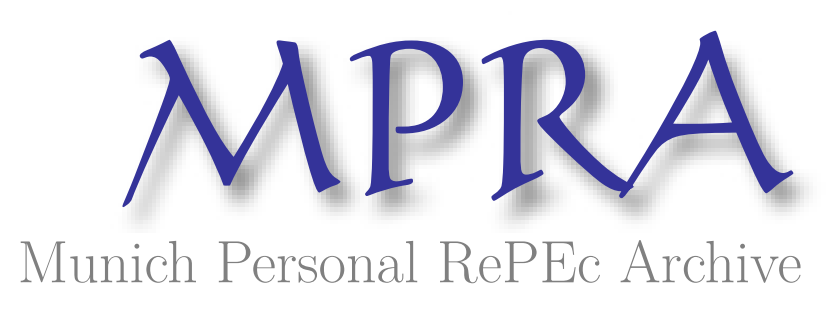

\title{
A Model for the Valuation of Assets with Liquidity Risk
}

Nauta, Bert-Jan

18 September 2016

Online at https://mpra.ub.uni-muenchen.de/92493/

MPRA Paper No. 92493, posted 04 Mar 2019 13:06 UTC 


\title{
A Model for the Valuation of Assets with Liquidity Risk
}

\author{
Bert-Jan Nauta
}

September 18, 2016

\begin{abstract}
This paper describes a model for the valuation of assets on a bank balance sheet with liquidity risk. The new feature of this model is that it explicitly incorporates the funding term of an asset. The inclusion of the funding term is important since it determines the expected liquidation loss. By minimizing the sum of the expected liquidation loss and funding costs the optimal funding term and value of the asset can be determined. This paper applies the model to single cash flows, loans, bonds, and derivatives. Also, the calibration to LIBOR basis spreads is discussed.
\end{abstract}

\section{Introduction}

Liquidity risk is one of the main risks for banks. Indeed, one of a bank's functions is to transform illiquid assets, such as long-term loans, into liquid liabilities, such as short-term deposits. Since the recent crisis, more focus has been placed on liquidity risk management by banks, partly enforced by regulations such as Basel 3. Despite the recent efforts to improve liquidity risk management, the inclusion of liquidity risk in the valuation of assets is still an area that is less well-developed. Most standard textbooks on derivatives pricing or loan pricing do not discuss the impact of liquidity risk on pricing (Hull 2008). Or they include liquidity risk based on benchmarking to other assets whose prices are known in the marketplace (Damodaran 2002), lacking a consistent approach across different assets on a bank balance sheet. The purpose of this paper is exactly to develop such a consistent approach. The paper applies the liquidity risk model proposed in (Nauta 2015b) to the most important assets on a bank balance sheet. 
The liquidity risk model in this paper assumes that at an uncertain (modeled as random) time in the future some of the funding markets of a bank freeze, which can be a bank-specific or a market-generic event. The duration of the event is uncertain as well (also modeled as random). If the funding of an asset is shorter than the duration of the event, the asset will need to be liquidated when the funding cannot be rolled over. The liquidation of the asset will lead to a loss depending on the liquidity of the asset. Since longer term funding is more expensive than shorter-term funding, there are two opposing forces at work. Funding costs push to shorter terms for funding whereas liquidity risk pushes to longer terms. The optimum depends on the liquidity (and other characteristics) of the asset. The value of the asset includes the funding costs at the optimal funding term and the residual liquidity risk.

This model is extremely simple as it reduces all complexities of liquidity risk to the timing and duration of a liquidity stress event. The simplicity of this model is similar to that of reduced form models for credit risk that also ignore all complexities of a default and simply model a random default time. The main benefit of this simplicity is that it allows for easier valuation and calibration than a more involved model.

Despite the simplicity of above liquidity risk model, it is rich enough to include and determine an optimal funding term for an asset.

The motivation for this work comes from the question how banks should include liquidity risk in their valuation of assets. Nevertheless, the resulting model is independent of specifics of the holder of the assets. It can be equally well an insurance company, asset manager, or hedge fund. Therefore the model and the results presented in the paper go beyond the application to banks only. Nevertheless, we will continue to call the holder of the assets a bank just to keep it specific.

In recent years, some papers have appeared that develop models to include funding costs in the valuation of derivatives (Piterbarg 2010). These investigations have lead to the introduction of a funding valuation adjustment (FVA). FVA is being reported by some of the major banks nowadays. However, there are a few concerns: 1) Most (if not all) valuation approaches consider the risk of an asset and then determine the price of this risk, for example through replication in a Black-Scholes type of valuation of derivatives. However, funding costs are not hedging costs to hedge a risk. 2) Funding costs are the same for liquid and illiquid assets. In particular, the value of an asset that is as good as cash would incur a non-zero FVA leading to inconsistencies (Nauta 2015b). 3) It is unclear what the funding costs are that should be included. These could be bank-specific funding costs or some market consensus funding costs (Becker and Sherif 2015). 
By developing a valuation methodology for assets with liquidity risk, we believe these concerns can be addressed.

\section{Discount factor of a single cash flow}

\subsection{Definitions}

The first step is to define the value of an asset including liquidity risk. We start with the simplest case of an asset that pays 1 at time $T$.

Following (Nauta 2015a) we use the following definition of liquidity risk: Liquidity risk is the risk for an event to occur that would force a bank to liquidate some of its assets. We will call such an event a liquidity stress event (LSE).

The valuation of assets with liquidity risk cannot ignore the funding strategy. In particular, the funding term is important. We consider funding strategies where the asset is funded up to its liquidity horizon $t_{\text {liq }}$ and this funding is rolled over continuously until the asset is funded to maturity. Therefore, we first define the value of the asset at time 0 depending on the funding term $V_{0}\left(t_{\text {liq }}\right)$ through

$$
\mathbb{E}[\text { Pay-off }(T)]=V_{0}\left(t_{\text {liq }}\right) \operatorname{Accrual}_{t_{\text {liq }}}(0, T)
$$

This relation states that the value of the asset at maturity should equal the value of the liability at maturity in expectation. Therefore, in expectation, the equity does not increase or decrease. Under the assumption that liquidity risk can be replicated the expectation in (2.1) would be an expectation under the pricing measure. However, replication of liquidity risk is impossible or at least extremely difficult in practice. Therefore we consider in (2.1) the real world expectation.

A generalization of (2.1) takes an expectation on the r.h.s. as well. However here we consider deterministic interest rates in which case the definition (2.1) is sufficient.

The accrual factor represents the accrued interest on the liability that funds the asset. The accrual factor depends on the term of the funding entered, which is assumed to be continuously rolled over until maturity. Since we assume deterministic interest rates the accrual factor of the rolled-over funding can be expressed as

$$
\begin{aligned}
\operatorname{Accrual}_{t_{\text {liq }}}(0, T)= & \left\{\prod_{i=0}^{N-1}\left[1+t_{\text {liq }} L_{t_{\text {liq }}}\left(0, i t_{\text {liq }},(i+1) t_{\text {liq }}\right)\right]\right\} \\
& \times\left[1+\left(T-N t_{\text {liq }}\right) L_{t_{\text {liq }}}\left(0, N t_{\text {liq }}, T\right)\right]
\end{aligned}
$$


where $N$ is an integer such that $N \times t_{\text {liq }} \leq T<(N+1) \times t_{\text {liq. }}$. The rate $L_{t_{\text {liq }}}\left(t, t_{1}, t_{2}\right)$ is the time $t$ value of the forward rate from $t_{1}$ to $t_{2}$ with index $t_{\text {liq }}$. The index $t_{\text {liq }}$ indicates that the reference rate is the unsecured (marginal) funding rate with tenor $t_{\text {liq }}$.

As suggested by the notation, in practice we will think of $L_{t_{\text {liq }}}\left(t, t_{1}, t_{2}\right)$ as forward LIBOR rates. In section 2.5 we will discuss the difference between LIBOR rates and the marginal funding rate and consequences.

$L_{t_{\text {liq }}}\left(t, t_{1}, t_{2}\right)$ represents the forward rates of a curve with index $t_{\text {liq }}$. Typical curves are the OIS discount curve that would be represented by $t_{\text {liq }}=$ 1 day and 1 month, 3 months, 6 months, and 12 months index curves with resp. $t_{\text {liq }}=1 \mathrm{M}, 3 \mathrm{M}, 6 \mathrm{M}, 12 \mathrm{M}$. The multiple curves framework is described in e.g. (Ametrano and Bianchett 2013, Fries 2013).

The value is defined as the optimum over possible funding terms:

$$
V_{0}=\sup _{0<t_{\text {liq }} \leq T} V_{0}\left(t_{\text {liq }}\right)
$$

In practice, the liquidity horizon (and funding term) will have a minimum possible value $\Delta t$. We will use a minimum of one day, but other choices are possible. With a minimum for the funding term the supremum can be replaced by a maximum

$$
V_{0}=\max _{\Delta t<t_{\text {liq }} \leq T} V_{0}\left(t_{\text {liq }}\right)
$$

The optimal funding term $t_{\text {liq }}^{\text {optimal }}$ is defined through the relation

$$
V_{0}\left(t_{\text {liq }}^{\text {optimal }}\right)=V_{0}
$$

This definition of value where the bank optimizes the funding term is akin to the valuation of an American option where the holder is assumed to optimize its exercise. An alternative definition of value that is commonly used in models of incomplete markets is the value of a hedge portfolio that minimizes the residual risk (such as the traditional minimum variance criterion). Such a definition is less applicable to the model presented here since the optimum will always be a term funding equal to maturity of the asset independent of whether it is a liquid or illiquid asset. Hence the result will be term funding, and the value of liquid and illiquid asset would be the same. The optimization of the value with respect to the funding term leads to a value and optimal funding term dependent on the liquidity of the asset as we will see later. 


\subsection{Liquidity Risk Model}

We model the occurrence and duration of LSEs through independent random processes. The number of LSEs is assumed to follow a Poisson process. For our model we only need to model the occurrence of the first LSE whose time of occurrence $\tau$ follows an exponential distribution with intensity $\lambda$.

$$
\tau \sim \rho_{\exp }(\lambda ; \tau)=\lambda e^{-\lambda \tau}
$$

The duration of an LSE $t_{\text {event }}$ is modeled as random as well, and we assume it follows a lognormal distribution.

$$
t_{\text {event }} \sim \rho_{\mathrm{LN}}\left(\mu, \sigma ; t_{\text {event }}\right)=\frac{1}{t_{\text {event }} \sigma \sqrt{2 \pi}} e^{-\left(\log \left(t_{\text {event }}\right)-\mu\right)^{2} / 2 \sigma^{2}}
$$

When an LSE occurs, the bank may be forced to liquidate some of its assets. In our model an asset will be liquidated if its funding term is too short to "survive" the LSE: $t_{\text {liq }}<t_{\text {event }}$. When the asset is liquidated in an LSE it will be sold at a discount. This can be modeled by introducing an effective pay-off

$$
\begin{array}{rlr}
\text { Effective Pay-off } & =1 & \text { at time } T \text { if } \tau+t_{\text {liq }} \geq T \\
& =V_{\text {rf }}\left(\tau+t_{\text {liq }}\right) L V\left(t_{\text {event }}, t_{\text {liq }}\right) \quad \text { at time } \tau+t_{\text {liq }} \text { if } \tau+t_{\text {liq }}<T
\end{array}
$$

The risk-free value $V_{\mathrm{rf}}(t)$ is the value of the cash flow without liquidity risk

$$
V_{\mathrm{rf}}(t)=D F_{\mathrm{ON}}(t, T)=e^{-r_{\mathrm{ON}}(T-t)}
$$

where we have used that without liquidity risk the asset can be funded on an overnight $(\mathrm{ON})$ basis and $D F_{\mathrm{ON}}(t, T)$ is the discount factor based on the overnight rate

$$
D F_{\mathrm{ON}}(t, T)=1 / \operatorname{Accrual}_{t_{O N}}(t, T) \text {. }
$$

The fraction $L V\left(t_{\text {event }}, t_{\text {liq }}\right)$ denotes the liquidation value as a fraction of the risk-free value. It is a modeling choice to define $L V$ as a fraction of the riskfree value instead of the value including liquidity risk. This choice somewhat simplifies calculations, and avoids additional choices involved in defining the risky value during an LSE. The liquidation value $L V$ should be equal to 1 when the funding term is larger than the duration of the LSE and we expect it to decrease as a function of $t_{\text {event }}-t_{\text {liq }}$ until some minimum value $L V_{\text {min }}$ is reached. A simple piece-wise linear function that models this behavior is

$$
L V\left(t_{\text {event }}, t_{\text {liq }}\right)=\max \left(1-c\left(t_{\text {event }}-t_{\text {liq }}\right) I_{t_{\text {event }}>t_{\text {liq }}}, L V_{\text {min }}\right)
$$


where $L V_{\min }$ is the minimum liquidation value $0 \leq L V_{\min } \leq 1$ and $c>0$.

To apply the definition (2.1) the pay-off needs to be defined at a single time $T$. Therefore we define the pay-off at time $T$ from the effective pay-off $(2.8)$ as

$$
\begin{aligned}
\operatorname{Pay-off}(T) & =1 & & \text { if } \tau+t_{\text {liq }} \geq T \\
& =V_{\text {rf }}\left(\tau+t_{\text {liq }}\right) L V\left(t_{\text {event }}, t_{\text {liq }}\right) \text { Accrual }_{t_{\text {liq }}}\left(\tau+t_{\text {liq }}, T\right) & & \text { if } \tau+t_{\text {liq }}<T
\end{aligned}
$$

The assumption here is that the resultant cash amount from the liquidation at time $\tau+t_{\text {liq }}$ will be used to reduce the funding. Therefore the accrual factor is based on the index $t_{\text {liq }}$ instead of e.g. the ON index.

\subsection{Derivation}

The definition (2.1) can be rewritten as

$$
V_{0}\left(t_{\text {liq }}\right)=\mathbb{E}[\operatorname{Pay}-\text { off }(T)] / \text { Accrual }_{t_{\text {liq }}}(0, T)
$$

By defining the $t_{\text {liq }}$ index discount factor

$$
D F_{t_{\text {liq }}}(0, T)=1 / \text { Accrual }_{t_{\text {liq }}}(0, T)
$$

and inserting the pay-off (2.12) in (2.13) the funding-term dependent value can be expressed as

$$
\begin{aligned}
V_{0}\left(t_{\text {liq }}\right) & =D F_{t_{\text {liq }}}(0, T) \mathbb{P}\left(\tau+t_{\text {liq }} \geq T\right) \\
& +\mathbb{E}\left[D F_{t_{\text {liq }}}\left(0, \tau+t_{\text {liq }}\right) V_{\text {rf }}\left(\tau+t_{\text {liq }}\right) I_{\tau+t_{\text {liq }}<T}\right] \mathbb{E}\left[L V\left(t_{\text {event }}, t_{\text {liq }}\right)\right]
\end{aligned}
$$

Here we have used that the random processes for the duration of the event $t_{\text {event }}$ and time of the event $\tau$ are independent and the relation

$$
D F_{t_{\text {liq }}}(0, T) \text { Accrual }_{t_{\text {liq }}}\left(\tau+t_{\text {liq }}, T\right)=D F_{t_{\text {liq }}}\left(0, \tau+t_{\text {liq }}\right)
$$

The expectation value of the product of the discount factor for index $t_{\text {liq }}$ and risk-free value at the liquidation date $\tau+t_{\text {liq }}$ can be calculated as

$$
\begin{aligned}
\mathbb{E}\left[D F_{t_{\mathrm{liq}}}\left(0, \tau+t_{\mathrm{liq}}\right) V_{\mathrm{rf}}\left(\tau+t_{\mathrm{liq}}\right) I_{\tau+t_{\mathrm{liq}}<T}\right] & =\mathbb{E}\left[e^{-r_{t_{\mathrm{liq}}}\left(\tau+t_{\mathrm{liq}}\right)} e^{-r_{\mathrm{ON}}\left(T-\left(\tau+t_{\mathrm{liq}}\right)\right)} I_{\tau+t_{\mathrm{liq}}<T}\right] \\
& =e^{-r_{t_{\mathrm{liq}}} T} e^{\left(r_{t_{\mathrm{liq}}}-r_{\mathrm{ON}}\right)\left(T-t_{\mathrm{liq}}\right)} \\
& \times \frac{\lambda}{\lambda+r_{t_{\mathrm{liq}}}-r_{\mathrm{ON}}}\left[1-e^{-\left(\lambda+r_{t_{\mathrm{liq}}}-r_{\mathrm{ON}}\right)\left(T-t_{\mathrm{liq}}\right)}\right]
\end{aligned}
$$


Here we have introduced the continuously compounded discount rate

$$
r_{t_{\text {liq }}}(t)=-\log \left(D F_{t_{\text {liq }}}(0, t)\right) / t
$$

Note that we have suppressed the $t$-dependence of $r_{t_{\text {liq }}}$ and $r_{\mathrm{ON}}$ in (2.17) to keep the equation readable. Combining (2.15) and (2.17) gives the result

$$
\begin{aligned}
V_{0}\left(t_{\text {liq }}\right) & =e^{-r_{t_{\text {liq }}} T} e^{-\lambda\left(T-t_{\text {liq }}\right)} \\
& +e^{-r_{t_{\text {liq }}} T} e^{\left(r_{t_{\text {liq }}}-r_{\mathrm{ON}}\right)\left(T-t_{\text {liq }}\right)} \\
& \times \frac{\lambda}{\lambda+r_{t_{\text {liq }}}-r_{\mathrm{ON}}}\left[1-e^{-\left(\lambda+r_{t_{\text {liq }}}-r_{\mathrm{ON}}\right)\left(T-t_{\text {liq }}\right)}\right] \mathbb{E}\left[L V\left(t_{\text {event }}, t_{\text {liq }}\right)\right]
\end{aligned}
$$

where we have suppressed the dependence of the rates on $T$. Note that this result assumes $T>t_{\text {liq }}$.

It is convenient to define a risky discount factor

$$
\begin{aligned}
D F_{\text {risky }}\left(t_{\text {liq }} ; 0, T\right) & =e^{-r_{t_{\text {liq }}}(T) T} e^{-\lambda\left(T-t_{\text {liq }}\right)^{+}} \\
& +e^{-r_{t_{\text {liq }}}(T) T} e^{\left(r_{t_{\text {liq }}}(T)-r_{\mathrm{ON}}(T)\right)\left(T-t_{\text {liq }}\right)^{+}} \frac{\lambda}{\lambda+r_{t_{\text {liq }}}(T)-r_{\mathrm{ON}}(T)} \\
& \times\left[1-e^{-\left(\lambda+r_{t_{\text {liq }}}(T)-r_{\mathrm{ON}}(T)\right)\left(T-t_{\mathrm{liq}}\right)^{+}}\right] \mathbb{E}\left[L V\left(t_{\text {event }}, t_{\text {liq }}\right)\right]
\end{aligned}
$$

where the notation (. $)^{+}$is used for the max-function $(x)^{+}=\max (x, 0)$. By using this max-function this risky discount factor is also valid in case $T<t_{\text {liq }}$.

The value of the single cash flow can be expressed in terms of the risky discount factor as

$$
V_{0}\left(t_{\text {liq }}\right)=D F_{\text {risky }}\left(t_{\text {liq }} ; 0, T\right)
$$

The expectation value of the liquidation value can be calculated explicitly and reads

$$
\begin{aligned}
\mathbb{E}\left[L V\left(t_{\text {event }}, t_{\text {liq }}\right)\right] & =N\left(\mu, \sigma ; \log \left(t_{\text {liq }}\right)\right) \\
& +\left(1+c t_{\text {liq }}\right) N\left(\mu, \sigma ; \log \left(t_{\text {liq }}\right), \log \left(t_{m}\right)\right) \\
& -c e^{\mu+\sigma^{2} / 2} N\left(\mu+\sigma^{2}, \sigma ; \log \left(t_{\text {liq }}\right), \log \left(t_{m}\right)\right) \\
& +L V_{\min }\left(1-N\left(\mu, \sigma ; \log \left(t_{m}\right)\right)\right)
\end{aligned}
$$

where $t_{m}=t_{\text {liq }}+\left(1-L V_{\min }\right) / c$, and $N$ is the cumulative normal

$$
\begin{aligned}
N\left(\mu, \sigma ; \log \left(t_{1}\right)\right) & =\int_{0}^{t_{1}} d t \rho_{\mathrm{LN}}(\mu, \sigma ; t) \\
N\left(\mu, \sigma ; \log \left(t_{1}\right), \log \left(t_{2}\right)\right) & =\int_{t_{1}}^{t_{2}} d t \rho_{\mathrm{LN}}(\mu, \sigma ; t)
\end{aligned}
$$

This completes the calculation of the funding term dependent value of a single cash flow with liquidity risk. 


\begin{tabular}{|l|c|c|}
\hline asset & $\mathrm{c}$ & $L V_{\min }$ \\
\hline liquid & 0.5 & 0.9 \\
\hline less liquid & 2 & 0.5 \\
\hline illiquid & 1000 & 0.0 \\
\hline
\end{tabular}

Table 1: Parameters for a liquid, less liquid and illiquid asset.

\subsection{Interpretation}

For the interpretation of the result in the previous section it is useful to expand the result $(2.19)$ in small $\left(r_{t_{\text {liq }}}-r_{\mathrm{ON}}\right) T$ and $\lambda T$. To first order the result is

$$
V_{0}\left(t_{\text {liq }}\right)=e^{-r_{\mathrm{ON}} T}\left\{1-\left(r_{t_{\text {liq }}}-r_{\mathrm{ON}}\right) T-\lambda\left(T-t_{\text {liq }}\right)\left[1-\mathbb{E}\left[L V\left(t_{\text {event }}, t_{\text {liq }}\right)\right]\right]\right\}
$$

The first term can be interpreted as the risk-free value of the single cash flow. The second term can be interpreted as (additional) funding costs

$$
F C=\left(r_{t_{\text {liq }}}-r_{\mathrm{ON}}\right) T
$$

The third term can be interpreted as liquidity costs

$$
L C=\lambda\left(T-t_{\text {liq }}\right)\left[1-\mathbb{E}\left[L V\left(t_{\text {event }}, t_{\text {liq }}\right)\right]\right]
$$

The definition of the value as the supremum of $V_{0}\left(t_{\text {liq }}\right)$ over funding terms, can be interpreted as minimizing the liquidity costs plus funding costs. To reduce liquidity costs, the funding term would need to increase, which increases funding costs. Hence, in some sense funding costs and liquidity costs can be viewed as two opposing forces: funding costs push to smaller funding terms and liquidity costs push to larger funding terms. The optimum depends on the liquidity of the asset as defined by the liquidation value $L V$.

To illustrate this mechanism we consider three cases: a liquid asset, a less liquid asset and an illiquid asset, all with a maturity of one year. The parameters for these three cases are shown in the table 1.

The liquidity risk model in this example is specified by the following parameters

$$
\begin{aligned}
& \lambda=80 \mathrm{bp} \\
& \mu=\log (0.5) \\
& \sigma=0.5
\end{aligned}
$$

The funding costs and liquidity costs are shown in figures 1, 2, and 3 as a function of the funding term. The funding costs are independent of the 


\begin{tabular}{|l|c|c|c|c|}
\hline asset & $t_{\text {liq }}^{\text {optimal }}$ & FC (in bp) & LC (in bp) & FC +LC (in bp) \\
\hline liquid & ON & 0 & 8 & 8 \\
\hline less liquid & 6 months & 25 & 7 & 32 \\
\hline illiquid & 9 months & 35 & 4 & 39 \\
\hline
\end{tabular}

Table 2: Optimal funding term, funding and liquidity costs for a liquid, less liquid and illiquid asset example.

liquidity of the asset and are the same in the three figures. The liquidity costs differ for the three assets. As expected the liquidity costs decrease with an increasing funding term. For the liquid asset we see that in this example the optimal funding term is $\mathrm{ON}$, as this minimizes the combined costs FC+LC. For the liquid asset the total costs at the optimal funding term consist of liquidity costs only and amounts to $8 \mathrm{bp}$. For the less liquid asset the optimal funding term is 6 months and the total costs are $32 \mathrm{bp}$. For the illiquid asset the optimal funding term is 9 months and the total costs are $39 \mathrm{bp}$. These results are summarized in the table 2 .

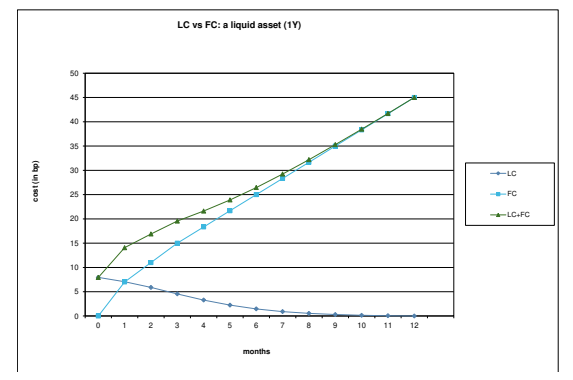

Figure 1: Funding costs and liquidity costs as a function of the funding term for a liquid asset.

\subsection{Funding strategies and funding rates}

The above definition of value (2.3) optimizes the value on a single funding term. More general funding strategies can be considered that will lead to a (slightly) higher value. That a single fixed funding term is not optimal among a set of general funding strategies may be seen as follows. Consider again the 1-year illiquid asset from Fig 3. We know from previous results that the optimal funding term is 9 months. The corresponding funding strategy is 


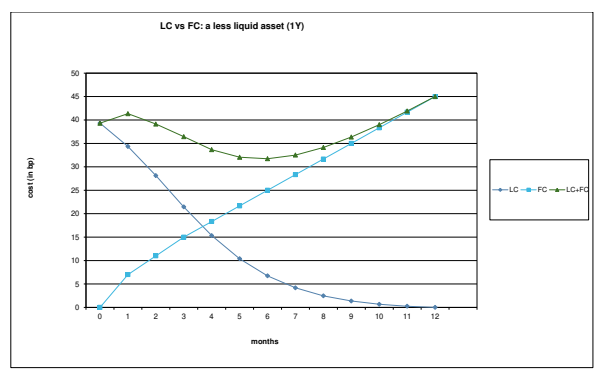

Figure 2: Funding costs and liquidity costs as a function of the funding term for a less liquid asset.

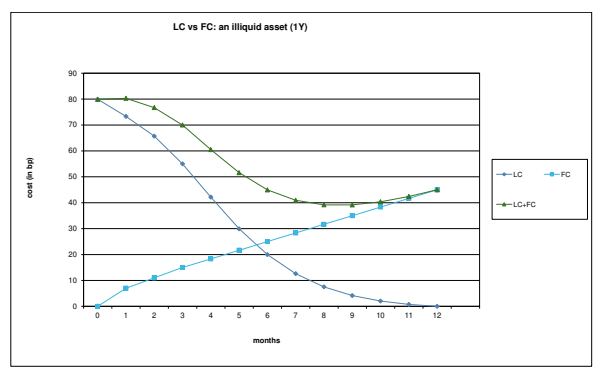

Figure 3: Funding costs and liquidity costs as a function of the funding term for an illiquid asset.

rolling over 9-month funding for three months (until 9 months to maturity). However considering an 11-month illiquid asset we find that the optimal funding term is 8 months. Hence, the optimal funding strategy when allowing for different funding terms through time is not a single fixed funding term.

In section 2.1 we mentioned that $L_{t_{\text {liq }}}\left(t, t_{1}, t_{2}\right)$ is the time $t$ value of the forward rate from $t_{1}$ to $t_{2}$ with index $t_{\text {liq }}$, where the index $t_{\text {liq }}$ indicates as reference rate the unsecured (marginal) funding rate with tenor $t_{\text {liq }}$. There are (at least) two choices for the reference rate:

1. unsecured marginal funding rate. This choice implies the assumption of market efficiency. It is not really necessary that the market is efficient, but rather the choice is to value assets as if the (funding) market is efficient. It can be shown that the marginal funding rate is equal to the risk-free rate (where risk-free means risk-free of market and credit risk, not liquidity risk) see e.g. (Burgard and Kjaer 2011; Hull and 
White 2014; Nauta 2015a).

We will assume that the marginal funding rate of a bank can be approximated by a LIBOR rate. In principle, the LIBOR rate is not free of credit risk, but studies show that the credit risk component is smaller than its liquidity risk component (Chang and Schlogl 2014). Since swaps on LIBOR rates with different tenors are quoted, these are a suitable practical approximation to marginal funding rates.

2. unsecured funding rate. In this case, one calculates a bank's internal value assuming that the bank's investors (liability holders) will not recognize the quality of the asset, which will lead to a bank-specific value. This value may be useful for bank's management or pricing.

\subsection{Discussion}

We have found that the risky discount factor for a single cash flow at time $T$ is given by $D F_{\text {risky }}\left(t_{\text {liq }}^{\text {optimal }} ; 0, T\right)$, see $(2.20)$. A useful approximation that gives an intuitive result is

$$
D F_{\text {risky }}\left(t_{\text {liq }}^{\text {optimal }} ; 0, T\right)=e^{-\left(r_{t_{\text {liq }}^{\text {optimal }}}+l\right) T}
$$

with the liquidity spread $l$ defined as

$$
l=\lambda \frac{T-t_{\mathrm{liq}}^{\text {optimal }}}{T}\left[1-\mathbb{E}\left[L V\left(t_{\text {event }}, t_{\text {liq }}^{\text {optimal }}\right)\right]\right]
$$

The approximation is accurate to first order in $\left(r_{t_{\text {liq }}^{\text {optimal }}}-r_{\mathrm{ON}}\right) T$ and $\lambda T$.

These approximate results are consistent with results in earlier work (Nauta 2015b). There, a model was used where a bank could liquidate a fraction $f$ so that $\lambda$ needs to be identified with $p \times f$ in that paper (there $p$ denotes the probability per annum of the occurrence of an LSE). Furthermore, multiple LSEs were allowed leading to differences in the detailed expressions.

It may be useful to compare (2.28) to the discount factor obtained in research concerning FVA, see e.g. (Piterbarg 2010) and (Burgard and Kjaer 2010)

$$
D F_{\text {funding }}(0, T)=e^{-\int_{0}^{T} r_{F}(t) d t}
$$

with $r_{F}(t)$ a bank's unsecured funding rate. The implicit assumption seems to be that an asset needs to be term-funded always. In particular, the liquidity of the asset does not affect the discount rate. 


\section{From portfolio valuation to asset valuation}

In the previous section, we have considered an asset in isolation. In reality, balance sheets contain multiple assets. In an LSE the bank can choose which assets it wants to sell. E.g. when it has some liquid assets and some illiquid assets the bank can liquidate the liquid assets first. Therefore it seems that the value can only be determined on a portfolio level and not on an asset level. However, we will argue that precisely because a bank can choose the order in which to liquidate assets, the valuation of a portfolio reduces to the valuation of its individual assets in this model.

Consider a particular asset in a portfolio with one other asset, namely cash. The cash is funded ON. The asset is funded with a term $t_{\text {liq. }}$. In an LSE after one day, the ON funding cannot be rolled over, and the cash is used to repay these ON loans. Once the duration of the LSE exceeds $t_{\text {liq }}$ the asset needs to be liquidated.

Now consider the asset is in a portfolio with an illiquid asset. We assume that the asset is again funded with a term $t_{\text {liq }}$ and that the illiquid asset is funded longer term. In this case, our particular asset is the first to be liquidated, but similar as in the previous example, this occurs when the duration of the LSE exceeds $t_{\text {liq }}$.

These two examples provide some intuition why the other assets in a portfolio may not affect the funding term and valuation of a particular asset.

To derive the valuation of multiple assets in a portfolio, we need to specify the model further. We make three additional assumptions.

1. For each asset, there is a separate funding source with its funding term. As in the previous section we limit the funding strategy to funding with one fixed funding term that is continuously rolled over until the funding term equals maturity of the asset.

2. The bank liquidates the assets to minimize liquidation losses. This means it will liquidate the most liquid assets first.

3. The loss resulting from the liquidation of one asset does not trigger liquidation of other assets. The other assets are only liquidated when the duration of the LSE exceeds their funding terms.

For the derivation consider a portfolio of two assets $A_{1}$ and $A_{2}$. For simplicity we assume both assets have the same maturity $T$. The value of the portfolio at time 0 depending on two funding terms $V_{0}\left(t_{\text {liq }}^{1}, t_{\text {liq }}^{2}\right)$ is defined similarly as for the single asset (2.1)

$$
\mathbb{E}\left[\text { Pay-off }_{\text {portf }}(T)\right]=V_{0}\left(t_{\text {liq }}^{1}, t_{\text {liq }}^{2}\right) \text { Accrual }_{t_{\text {liq }}^{1}, t_{\text {liq }}^{2}}(0, T)
$$


The accrual factor reflects that that funding is provided for $A_{1}$ and $A_{2}$ with different funding terms $t_{\text {liq }}^{1}$ and $t_{\text {liq }}^{2}$ (assumption 1)

$$
\operatorname{Accrual}_{t_{\mathrm{liq}}^{1}, t_{\mathrm{liq}}^{2}}(0, T)=w \operatorname{Accrual}_{t_{\mathrm{liq}}^{1}}(0, T)+(1-w) \operatorname{Accrual}_{t_{\mathrm{liq}}^{2}}(0, T)
$$

where we fix the weight $w$ by

$$
w=\frac{V_{A_{1}, 0}\left(t_{\text {liq }}^{1}\right)}{V_{A_{1}, 0}\left(t_{\text {liq }}^{1}\right)+V_{A_{2}, 0}\left(t_{\text {liq }}^{2}\right)}
$$

where $V_{A_{1}, 0}$ and $V_{A_{2}, 0}$ denote the values of the assets $A_{1}$ and $A_{2}$ as calculated in $(2.19)$.

Now we will assume that asset $A_{1}$ is more liquid than $A_{2}$. In particular we assume

$$
L V_{A_{1}}\left(t_{\text {event }}, t_{\text {liq }}\right) \geq L V_{A_{2}}\left(t_{\text {event }}, t_{\text {liq }}\right)
$$

for all $t_{\text {event }}$ and all $t_{\text {liq }}$. Then rational liquidation by the bank means it will liquidate $A_{1}$ before it liquidates $A_{2}$, since there is some probability it does not need to liquidate the second asset. The consequence of this strict order in liquidation (assumption 2) and the assumption that liquidation of one asset does trigger another liquidation (assumption 3) is that the pay-off of the portfolio is a sum of the pay-offs of the assets

$$
\text { Pay-off }_{\text {portf }}(T)=\text { Pay-off }_{A_{1}}(T)+\text { Pay-off }_{A_{2}}(T)
$$

It follows from a straightforward calculation that the funding term dependent portfolio value is a sum of the asset values

$$
\begin{aligned}
V_{0}\left(t_{\text {liq }}^{1}, t_{\text {liq }}^{2}\right) & =\mathbb{E}\left[\text { Pay-off }_{\text {portf }}(T)\right] / \operatorname{Accrual}_{t_{\text {liq }}^{1}, t_{\text {liq }}^{2}}(0, T) \\
& =\left(\mathbb{E}\left[\text { Pay-off } A_{A_{1}}(T)\right]+\mathbb{E}\left[\text { Pay-off }_{A_{2}}(T)\right]\right) / \text { Accrual }_{t_{\text {liq }}^{1}, t_{\text {liq }}^{2}}(0, T) \\
& =V_{A_{1}, 0}\left(t_{\text {liq }}^{1}\right)+V_{A_{2}, 0}\left(t_{\text {liq }}^{2}\right)
\end{aligned}
$$

We note that the combined optimization over $t_{\text {liq }}^{1}$ and $t_{\text {liq }}^{2}$ of the portfolio value reduces to separate optimizations of the individual asset values. Hence the value of the portfolio is the sum of values of the two assets

$$
V_{\text {portf }}=V_{A_{1}}+V_{A_{2}}
$$

where the value of these assets are defined as in (2.3) in the previous section.

This derivation can easily be extended to more assets and different maturities. It is an open question if the decomposition of the portfolio value into individual asset values also holds when general funding strategies, as discussed in subsection 2.5, are allowed. Another open question is if the strict 
order in liquidity (3.4) can be relaxed. Both questions are left for future research.

Now that we have established that the value of a portfolio consists of a sum of values of the individual assets, it makes sense to study the latter. In the previous section the value of a simple cash flow was derived. The rest of the paper is devoted to applying the model to some other important assets on a bank balance sheet.

\section{Valuation of a loan}

In this section we apply the model to the valuation of a loan. The loan is a collection of incoming cash flows

$$
C F_{i} \text { at time } t_{i}
$$

with $C F_{i}>0$ for all $i$. The index $i=1, \ldots, N$ labels the cash flows in increasing time $t_{i}<t_{i+1}$, and $t_{N}=T$ equals the maturity of the loan. We consider only simple loans; the cash flows are not contingent on other events. The reason to consider the loan separately is that if the loan needs to be liquidated this implies liquidation of all remaining cash flows jointly.

Here we derive the value of a loan assuming the funding strategy consists of funding the loan with a single funding term. Consistent with the approach for a single cash flow (2.12) we define the pay-off at maturity $T$ as

$$
\begin{aligned}
\operatorname{Pay-off}(T) & =\sum_{i=1}^{N} C F_{i} \operatorname{Accrual}_{t_{\mathrm{liq}}}\left(t_{i}, T\right) I_{\tau+t_{\mathrm{liq}} \geq t_{i}} \\
& +V_{\mathrm{rf}}\left(\tau+t_{\mathrm{liq}}\right) L V\left(t_{\text {event }}, t_{\text {liq }}\right) \text { Accrual }_{t_{\mathrm{liq}}}\left(\tau+t_{\mathrm{liq}}, T\right) I_{\tau+t_{\mathrm{liq}}<T}
\end{aligned}
$$

Inserting this pay-off in (2.13) gives

$$
\begin{aligned}
V_{0}\left(t_{\text {liq }}\right) & =\sum_{i=1}^{N} D F_{t_{\text {liq }}}\left(0, t_{i}\right) C F_{i} \mathbb{P}\left(\tau+t_{\text {liq }} \geq t_{i}\right) \\
& +\mathbb{E}\left[D F_{t_{\text {liq }}}\left(0, \tau+t_{\text {liq }}\right) V_{\text {rf }}\left(\tau+t_{\text {liq }}\right) I_{\tau+t_{\text {liq }}<T}\right] \mathbb{E}\left[L V\left(t_{\text {event }}, t_{\text {liq }}\right)\right]
\end{aligned}
$$

Calculations similar to the single cash flow case yield the expected result

$$
V_{0}\left(t_{\text {liq }}\right)=\sum_{i=1}^{N} D F_{\text {risky }}\left(t_{\text {liq }} ; 0, t_{i}\right) C F\left(t_{i}\right)
$$

Hence the funding-term dependent value of the loan is a simple sum of the funding-term dependent values of the individual cash flows. 
However, this simple decomposition does not hold for the value, since the optimal funding term of individual cash flows may differ. E.g. in the example in section 2.4 of an illiquid loan the 1-year cash flow will have an optimal funding term of 9 months whereas a 6 -month cash flow will have an optimal funding term of 6 months or less. Hence based on a single funding term the value of a loan is not equal to the sum of the values of its cash flows

$$
V_{0}^{\text {loan }} \neq \sum_{i=1}^{N} V_{0}^{\mathrm{SCF}}
$$

Note that this is not simply a consequence of the assumption of a single funding term. With multiple funding terms, the loan needs to be liquidated after shortest funding cannot be rolled over in an LSE. Although one could assume that a fraction of the loan can be liquidated, it is not possible to liquidate a single cash flow out of a loan. Therefore, the general conclusion holds, that in this model for valuation with liquidity risk the value of a loan is not a simple sum of the values of the individual cash flows.

The difference in value between the sum of simple cash flows and the loan is however small. Consider as an example an illiquid loan with monthly payments of $5 \%$ per annum, notional 100, and maturity 1 year. For the calculation of the value we have assumed a zero risk-free discount rate; funding spreads are the same as in the examples in fig 1,2 , and 3 . The result is

\begin{tabular}{|c|c|c|}
\hline & loan & sum of SCFs \\
\hline value & 104.5988 & 104.6000 \\
\hline
\end{tabular}

The difference of 0.0012 is small indeed.

\section{Valuation of a bond}

The difference between a loan and a bond for the purpose of this paper is that a bond may generate liquidity through a repurchase agreement (repo). Hence, in the case of an LSE whose duration is longer than the funding term of the bond the bond may not have to be liquidated, but instead, can be repoed. For this reason, we consider bonds separately.

\subsection{Repo transaction instead of liquidation}

In a repo transaction, the bank will sell the bond and agree to buy it back at a later date. The cash received from the sale of the bond will not be the full value of the bond, but a haircut will be applied. Therefore, if the funding 
of a bond cannot be rolled over during an LSE, a repo transaction will only partly provide the cash required. Therefore, it is natural to consider two funding terms:

$$
\begin{aligned}
& t_{\text {liq }}^{\text {bond }} \text { for } B(1-h) \\
& t_{\text {liq }}^{\text {haircut }} \text { for } B h
\end{aligned}
$$

where $B$ denotes the value of the bond and $h$ the haircut in an LSE. The idea is that the funding of the haircut is longer term so that a repo transaction provides exactly the required cash amount when the shorter term $t_{\text {liq }}^{\text {bond }}$ needs to be refinanced.

The funding term $t_{\text {liq }}^{\text {bond }}$ depends on the liquidity of the repo transaction, but, if the repo market functions in an LSE for the relevant bond, this should be a short term. In any case, this part can be funded through repo transactions. For the valuation of the bond, the funding term of the haircut is more interesting. Using the same approximation as in (2.25) the funding costs become

$$
F C=h\left(r_{t_{\text {liq }}^{\text {haircut }}}-r_{\mathrm{ON}}\right) T .
$$

The main difference between the funding costs of a cash flow (2.25) and the bond, is exactly that only the funding costs of the haircut are included, which explains the factor $h$. The expected liquidation costs apply to the full bond. Therefore the liquidity costs are given by the usual expression

$$
L C=\lambda\left(T-t_{\text {liq }}\right)\left[1-\mathbb{E}\left[L V\left(t_{\text {event }}, t_{\text {liq }}^{\text {haircut }}\right)\right]\right]
$$

In Fig 4 the funding costs for a bond are shown. The bond is specified by the "less liquid" parameters in table 1 and has a maturity of 10 years. The liquidity risk model parameters are the same as in (2.27). The haircut is assumed to be 20\%, which is why the funding costs in Fig 4 are $20 \%$ of the funding costs in the examples in Fig 2. We see that the optimal funding term for the haircut is 12 months in this example. The funding costs plus liquidity costs add to 11 bp per annum.

Table 3 summarizes the optimal funding term for the haircut and funding and liquidity costs for different haircut percentages.

\section{Valuation of uncollateralized derivatives}

To apply the model to uncollateralized derivatives the main complication is that the value of the derivative at future times is unknown. This means that it is unknown what the potential loss due to liquidation in an LSE is, or what 


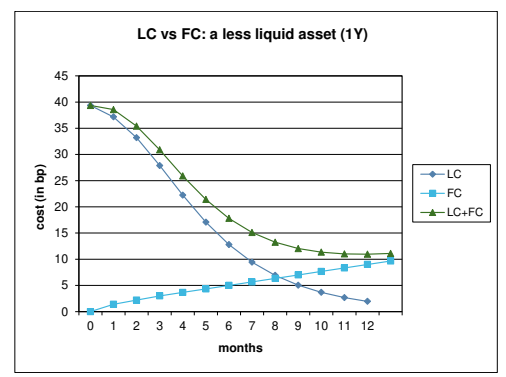

Figure 4: Funding costs and liquidity costs per annum for a 10-year bond with repo market. This bond is "less liquid" as defined in table 1.

\begin{tabular}{|l|c|c|c|c|}
\hline haircut & optimal $t_{\text {liq }}^{\text {haircut }}$ & FC (in bp) & LC (in bp) & FC +LC (in bp) \\
\hline $5 \%$ & 16 & 3 & 0 & 3 \\
\hline $10 \%$ & 14 & 5 & 1 & 6 \\
\hline $15 \%$ & 13 & 7 & 1 & 9 \\
\hline $20 \%$ & 12 & 9 & 2 & 11 \\
\hline $25 \%$ & 11 & 10 & 3 & 13 \\
\hline $30 \%$ & 10 & 11 & 4 & 15 \\
\hline
\end{tabular}

Table 3: Optimal funding term of the haircut, funding and liquidity costs per annum for a bond at different haircut percentages. The bond is of the "less liquid" type as specified in table 1 and has a maturity of 10 years.

the required funding is. Another complication is that many derivatives can take positive and negative values, such as a swap. For such derivatives, the liquidation loss in an LSE is not simply proportional to its value as for loans and bonds. We use the most simple extension and define the liquidation loss for derivatives as

$$
\text { liquidation loss }=(M t M)^{+}(1-L V) \text {, }
$$

but other choices are possible yielding different models.

It is useful to consider first the liquidation costs when funding is ON and then generalize to longer funding terms.

\subsection{ON funding}

In case of ON funding liquidation takes place a day after an LSE occurs. If we ignore this one day delay (and assume the value does not jump in this 
one day) the liquidation costs are

$$
L C=\mathbb{E}\left[\int_{0}^{\infty} d t D F_{\mathrm{ON}}(0, t)(M t M(t))^{+} \rho_{\mathrm{exp}}(\lambda ; t)\left(1-L V\left(t_{\mathrm{event}}, t_{\mathrm{ON}}\right)\right)\right]
$$

where MtM denotes the mark-to-market of the derivative. This MtM value excludes liquidity risk. It is the equivalent to the value $V_{\mathrm{rf}}$ used in (2.8).

Assuming independence of the duration of the LSE and the MtM, the expression (6.2) can be simplified as

$$
L C=\int_{0}^{\infty} d t D F_{\mathrm{ON}}(0, t) E E(t) \rho_{\mathrm{exp}}(\lambda ; t)\left(1-\mathbb{E}\left[L V\left(t_{\mathrm{event}}, t_{\mathrm{ON}}\right)\right]\right)
$$

where the expected exposure $E E(t)$ is defined as

$$
E E(t)=\mathbb{E}\left[(M t M(t))^{+}\right]
$$

In a portfolio with multiple derivatives netting is possible. For credit risk, e.g. in the case of CVA, one considers so-called netting sets. A netting set is the set of transactions that can be netted in the case of default of the counterparty. For liquidity risk instead of netting sets, we should consider funding sets as discussed in (Albanese and Andersen 2014). To define a funding set consider that each uncollateralized derivative is hedged with a collateralized counterpart (in the same book). The funding set is then the set of derivatives where the collateral can be rehypothecated among the hedges. A funding set is in general much larger than a netting set and may contain all derivative transactions of a legal entity. We refer to (Albanese and Andersen 2014) for a more extensive discussion of funding sets.

For equations (6.2), (6.3) and (6.4) this means the MtM should be interpreted as MtM of a funding set.

The expression for LC (6.3) is similar to expressions for CVA. To get from (6.3) to an expression for CVA the probability of an LSE should be replaced by probability of default, the expected loss in an $\operatorname{LSE}\left(1-\mathbb{E}\left[L V\left(t_{\text {event }}, t_{\mathrm{ON}}\right)\right]\right)$ should be replaced by the LGD, and funding sets should be replaced by netting sets, as summarized below.

\begin{tabular}{|c|c|}
\hline LC & CVA \\
\hline$\rho_{\exp }(\lambda ; t) d t$ & $P D(t, t+d t)$ \\
\hline$\left(1-\mathbb{E}\left[L V\left(t_{\text {event }}, t_{\mathrm{ON}}\right)\right]\right)$ & LGD \\
\hline Funding set & Netting set \\
\hline
\end{tabular}




\subsection{Funding term $>\mathrm{ON}$}

If $t_{\text {liq }}>t_{\mathrm{ON}}$ we need to consider more carefully what happens in an LSE. Funding just the MtM is insufficient if the MtM increases after the start of the LSE. This may seem strange since MtM going up results in profit and increase in equity, which automatically funds the increase in MtM.

To understand this paradox we define the value of (a funding set of) uncollateralized derivatives relative to its collateralized counterpart. The reference collateralized derivative has an idealized collateral agreement with a continuous posting of cash collateral, no thresholds, no initial margin, or other complicating features.

$$
\text { Uncoll }=\text { Coll }{ }^{\text {ideal }}-\mathrm{FC}-\mathrm{LC}
$$

Now it is convenient to consider an uncollateralized derivative hedged with its collateralized counterpart. Such a hedge should eliminate the market risk, but leaves credit risk and liquidity risk. Here we focus on liquidity risk. It is then clear that an MtM increase leads to an outgoing cash flow from the hedge (the collateralized derivative). Therefore, an MtM increase will lead to the liquidation of the derivative if only the MtM is funded. Hence, just funding the MtM is insufficient.

A well-known way to mitigate liquidity risk is through a liquidity buffer. A liquidity buffer consists of liquid assets that can be liquidated in a liquidity stress event to avoid forced liquidation of illiquid assets. For valuation purposes, it is sufficient to consider a liquidity buffer consisting of cash. The liquidity buffer is the extra cash amount that is funded to compensate for the volatility in the MtM.

In a similar approximation as in (2.25) the funding costs read

$$
\begin{aligned}
F C=\mathbb{E} & {\left[\int_{0}^{\infty} d t D F_{\mathrm{ON}}(0, t)\left(M t M(t)+L B_{t_{\mathrm{liq}}}(t)\right) e^{-\lambda t}\right.} \\
& \left.\times\left[L_{t_{\mathrm{liq}}}\left(0, t, t+t_{\mathrm{liq}}\right)-L_{t_{O N}}\left(0, t, t+t_{\mathrm{liq}}\right)\right]\right]
\end{aligned}
$$

Note that the liquidity buffer $L B$ has the same funding term as the MtM. Would the funding term be shorter the liquidity buffer would need to be liquidated after this short term in an LSE, and would not provide the buffer for MtM changes in an LSE.

The expression for the liquidity cost is

$$
L C=\mathbb{E}\left[\int_{0}^{\infty} d t D F_{\mathrm{ON}}\left(0, t+\tilde{t}_{\mathrm{liq}}\right)\left(M t M\left(t+\tilde{t}_{\mathrm{liq}}\right)\right)^{+} \rho_{\mathrm{exp}}(\lambda ; t)\left(1-L V\left(t_{\text {event }}, \tilde{t}_{\text {liq }}\right)\right)\right]
$$


in a similar approximation as in (2.26). Here $\tilde{t}_{\text {liq }}$ denotes the time the MtM is funded in an LSE. This is the minimum of the funding term $t_{\text {liq }}$ and the time that the liquidity buffer is depleted $t_{\text {hit }}$

$$
\tilde{t}_{\text {liq }}=\min \left(t_{\text {liq }}, t_{\text {hit }}\right)
$$

Since the latter is the same as a (hitting) time the barrier at level $L B$ is reached by the $M t M$, we call this a hitting time $t_{\text {hit }}$, defined as the minimal time when

$$
\operatorname{MtM}\left(\tau+t_{\mathrm{hit}}\right)=\operatorname{MtM}(\tau)+L B_{\text {liq }}(\tau)
$$

If we model the $M t M$ as a stochastic process, then $t_{\text {hit }}$ is a stochastic time.

\subsection{Estimating the optimal Liquidity Buffer}

To estimate the required size of the buffer a process for the MtM is required. We assume the MtM follows a standard Brownian motion:

$$
d M t M=\operatorname{vol} \times M t M_{0} \times d W
$$

where $M t M_{0}$ is the MtM at time 0 which is used to normalize the volatility. Hence "vol" denotes an annual volatility. The distribution of the hitting time $t_{\text {hit }}$ is then well-known

$$
\rho\left(t_{\text {hit }}\right)=\frac{a}{\sqrt{2 \pi} t_{\text {hit }}^{3 / 2}} e^{-a^{2} / 2 t_{\text {hit }}}
$$

with

$$
a=\frac{L B}{\operatorname{vol} \times M t M_{0}}
$$

When the MtM is large the optimal liquidity buffer $L B$ at a given time can be obtained by minimizing the costs:

$$
\begin{aligned}
\operatorname{costs} & =(M t M+L B)\left[L_{t_{\text {liq }}}\left(0, t, t+t_{\text {liq }}\right)-L_{O N}\left(0, t, t+t_{\text {liq }}\right)\right] \\
& +M t M \lambda \mathbb{E}\left[\left(1-L V\left(t_{\text {event }}, \tilde{t}_{\text {liq }}\right)\right)\right]
\end{aligned}
$$

for a given $M t M$. The condition that MtM is large (at time $t$ ) more precisely means that the probability of $M t M\left(t^{\prime}\right)<0$ for $t<t^{\prime}<t+t_{\text {liq }}$ is small.

As an example, we have performed the optimization over funding term and liquidity buffer jointly, for the same parameters and LIBOR spreads as used before. We have assumed furthermore that the set of uncollateralized 
derivatives is "illiquid" as defined in table 1. Assuming a volatility of $10 \%$ the result is

$$
\begin{aligned}
& t_{\text {liq }}^{\text {optimal }}=0.77 \\
& L B=16.9 \% \times M t M \\
& \text { costs }=57 \mathrm{bp} \times M t M
\end{aligned}
$$

These results may be compared to the results for an illiquid cash flow, see table 2. Note that the optimal funding term is close to the 9 months found in table 2 for illiquid assets. The associated costs were 39bp in that example. The process for the MtM (6.10) assumes an infinite maturity. Changing the maturity from 1-year to infinity in the example in table 2 results in the total costs of $51 \mathrm{bp}$. The remaining difference of $6 \mathrm{bp}$ comes from funding the buffer (6bp) and from extra liquidation losses (rounded 0bp) when the liquidity buffer is depleted before the optimal funding term.

By comparing the liquidity buffer with the volatility, the liquidity buffer can be expressed as a VaR. We note that the liquidity buffer is comparable to a $95 \%$ 1-year VaR of the funding set for our example

$$
L B \sim 1 \text {-year } \operatorname{VaR}_{95 \%}
$$

In table 4 results are shown for different volatilities.

Note that the funding costs and liquidity costs are defined relative to idealized collateralized derivatives. In particular, it is assumed that no initial margin needs to be posted for these collateralized derivatives. Green and Kenyon (Green and Kenyon 2014) discuss the inclusion of initial margin in the context of funding costs. The funding term for initial margin is discussed in (Nauta 2015c).

\section{On calibration}

There are two main options for the calibration of the model. A bank can choose to perform a firm-specific calibration or market-consistent calibration. In a firm-specific calibration, the resulting $\lambda$ indicates the probability that the bank will experience an LSE, which may be due to general market conditions or have some firm-specific cause. A firm-specific calibration will probably require some expert judgment as there are no traded instruments on the liquidity risk of specific firms. The model parameters could, for instance, be estimated from stress tests that the firm conducts.

A market-consistent calibration is almost by definition difficult, since it involves valuing illiquid instruments and comparing these values with market 


\begin{tabular}{|c|c|c|c|}
\hline vol & $t_{\text {liq }}^{\text {optimal }}$ & LB/MtM in \% & costs in bp \\
\hline 0.01 & 0.82 & 2.3 & 51 \\
0.02 & 0.81 & 4.3 & 52 \\
0.03 & 0.8 & 6.1 & 52 \\
0.04 & 0.8 & 7.9 & 53 \\
0.05 & 0.79 & 9.5 & 54 \\
0.06 & 0.79 & 11.1 & 55 \\
0.07 & 0.78 & 12.6 & 55 \\
0.08 & 0.78 & 14.1 & 56 \\
0.09 & 0.77 & 15.5 & 56 \\
0.1 & 0.77 & 16.9 & 57 \\
0.11 & 0.76 & 18.2 & 58 \\
0.12 & 0.76 & 19.5 & 58 \\
0.13 & 0.75 & 20.8 & 59 \\
0.14 & 0.75 & 22.1 & 59 \\
0.15 & 0.75 & 23.3 & 60 \\
0.16 & 0.74 & 24.5 & 60 \\
0.17 & 0.74 & 25.7 & 61 \\
0.18 & 0.74 & 26.9 & 61 \\
0.19 & 0.73 & 28.0 & 62 \\
0.2 & 0.73 & 29.1 & 62 \\
\hline
\end{tabular}

Table 4: The optimal funding term and liquidity buffer for different volatilities.

prices. However market prices for illiquid instruments are difficult to obtain since there are large bid-offer spreads, or prices are stale. In any case, a price cannot be immediately realized (otherwise the asset would be liquid) and, therefore, some uncertainty in the calibration remains.

In a market calibration, an important choice is what instruments to use for the calibration. Since liquidity risk is generic for all assets, in principle any asset class (or a combination) could be used, such as bonds, (governmentbacked) loans, or illiquid derivatives.

In this paper, we consider calibration to LIBOR spreads. LIBOR spreads are obvious candidates for the calibration of the model as LIBOR spreads are used for the estimation of funding costs as well. 


\subsection{Market calibration}

In this section we show the calibration results for a particular set of curves shown in Fig 5.

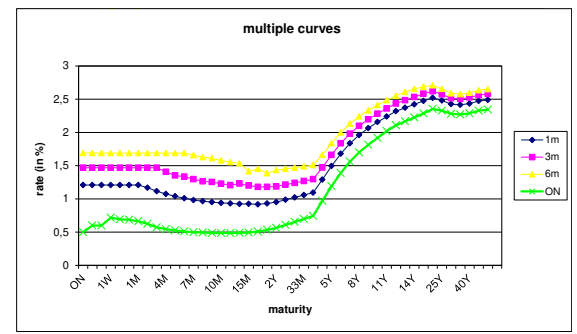

Figure 5: Interest rate curves used for calibration.

\begin{tabular}{|c|c|}
\hline parameter & value \\
\hline$\mu$ & $\log (0.5)$ \\
$\sigma$ & 0.5 \\
$\mathrm{c}$ & 1 \\
$L V_{\min }$ for $1 \mathrm{M}$ & $80 \%$ \\
\hline
\end{tabular}

We calibrate the intensity $\lambda$ and the liquidation value for $3 \mathrm{M}$ and $6 \mathrm{M}$. To be able to determine the intensity we fix the liquidation value of the $1 \mathrm{M}$ LIBOR at $80 \%$. The steps we follow are:

1. Calculate the intensity from the $\mathrm{ON}-1 \mathrm{M}$ spread for a liquidation value of $1 \mathrm{M}$ LIBOR at $80 \%$.

2. Calculate the liquidation value of $3 \mathrm{M}$ LIBOR. This is done by assuming a funding term of $\mathrm{ON}$ and $1 \mathrm{M}$. The optimal value (the lower LV) is used.

3. This is repeated for the liquidation value of $6 \mathrm{M}$ LIBOR. In this case funding terms of $\mathrm{ON}, 1 \mathrm{M}$ and $3 \mathrm{M}$ are considered.

Results are shown in figures 6 and 7 . It is encouraging to see that the calibrated LVs are relatively stable, although this is certainly no proof that the model captures the most important factors of liquidity risk. These results are consistent with the results in (Chang and Schlogl 2014), where liquidity costs calibration was tested for a large set of historical dates. 
Note that the probability of an LSE that starts at $280 \mathrm{bp}$ per annum is a direct consequence of the $80 \% \mathrm{LV}$ assumption for $1 \mathrm{M}$ LIBOR. All forward intensities are positive. There is a single outlier between 10 and 11 year, where the forward $\lambda$ is as low as $4 \mathrm{bp}$, but this seems a data issue.

The trend in the intensity is independent of specific assumptions. It is remarkable to see the low intensity beyond 20 years. Apparently, in this market, the probability of LSEs is expected to decrease significantly.

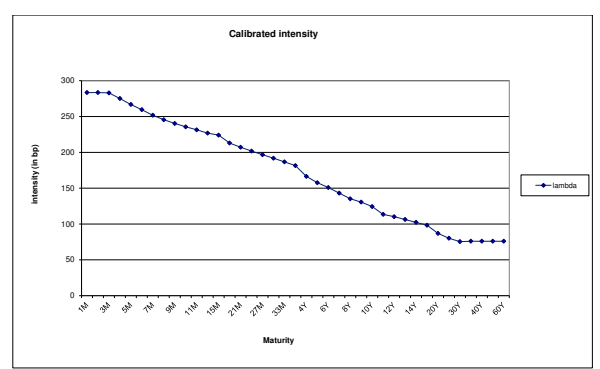

Figure 6: Calibrated intensity at different maturities.

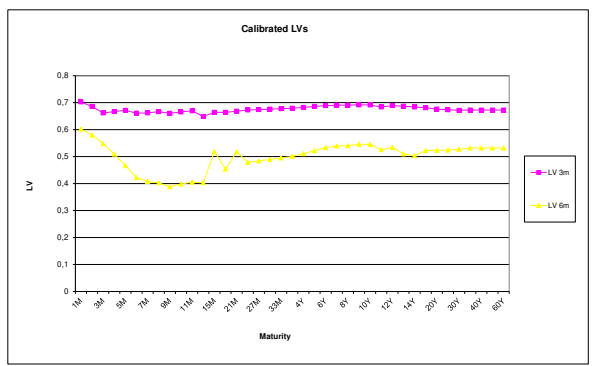

Figure 7: Calibrated liquidation values (LVs) for 3M and 6M LIBOR. (Note that the $1 \mathrm{M} \mathrm{LV}$ was fixed at $80 \%$.)

\section{Summary}

This paper defines the value of derivatives in a simple model for liquidity risk. In this model, liquidity risk leads to losses due to the liquidation of an asset when a liquidity stress event occurs, and the funding term of the 
asset is insufficient to 'survive' the LSE. By minimizing the sum of these expected liquidity losses and the funding costs the optimal funding term can be estimated. The optimal funding term determines the discount factor.

The application of the model to loans shows that the value of a loan cannot be decomposed as a sum of values of individual cash flows, although the difference is negligible in most practical circumstances.

For bonds for which there exists a repo market, instead of liquidation, a repo transaction can be used to generate liquidity in an LSE. However, the haircut needs to be funded separately. We have discussed how the funding term of the haircut can be determined.

For derivatives, a liquidity buffer is required to absorb volatility in the required funding due to MtM changes. Valuation of derivatives, therefore, requires optimization of the funding term and liquidity buffer together. We have calculated an example and show that the optimal liquidity buffer is approximately equal to the 1-year $95 \% \mathrm{VaR}$ in this case.

\section{Acknowledgements}

I would like to thank Michiel Lodewijk, Stratos Nikolakakis, Menno Dobber and Anna Motsartova for useful discussions. The first version of this paper was submitted when the author worked at RBS. The opinions expressed in this paper are those of the author and not necessarily those of RBS or the DNB.

\section{References}

C. Albanese and L. Andersen. Accounting for otc derivatives: Funding adjustments and the re-hypothecation opion. Available at SSRN: http://dx.doi.org/10.2139/ssrn.2482955, 2014.

F. M. Ametrano and M. Bianchett. Curves and term structure models: Definition, calibration and application of rate curves and term structure models. Available at SSRN: http://dx.doi.org/10.2139/ssrn.2194907, 2013.

L. Becker and N. Sherif. The black art of fva, part iii: a $\$ 4$ billion mistake? Risk magazine, 2015.

C. Burgard and M. Kjaer. Partial differential equation representations of derivatives with bilateral counterparty risk and funding costs. The Journal of Credit Risk, 2010.

C. Burgard and M. Kjaer. In the balance. Risk Magazine, 2011. 
Y. Chang and E. Schlogl. A consistent framework for modelling basis spreads in tenor swaps. Available at SSRN: http://dx.doi.org/10.2139/ssrn.2433829, 2014.

A. Damodaran. Investment Valuation: Tools and Techniques for Determining the Value of any Asset. John Wiley and Sons, second edition edition, 2002.

C. Fries. Everything you always wanted to know about multiple interest rate curve bootstrapping but were afraid to ask. Available at SSRN: http://dx.doi.org/10.2139/ssrn.2219548, 2013.

A. Green and C. Kenyon. Mva: Initial margin valuation adjustment by replication and regression. Available at SSRN: http://dx.doi.org/10.2139/ssrn.2432281, 2014.

J. Hull. Options, Futures and Other Derivatives. Prentice Hall, 7th edition edition, 2008.

J. Hull and A. White. Valuing derivatives: Funding value adjustments and fair value. Financial Analysts Journal, 2014.

B. J. Nauta. Liquidity risk, instead of funding costs, leads to valuation adjustments for derivatives and other assets. International Journal of Theoretical and Applied Finance, 2015a.

B. J. Nauta. Fva and liquidity risk. Available at SSRN: http://dx.doi.org/10.2139/ssrn.2373147, 2015b.

B. J. Nauta. Optimal funding term of posted collateral. Review of Financial Markets, 2015c.

V. Piterbarg. Funding beyond discounting: collateral agreements and derivatives pricing. Risk magazine, 2010. 